\title{
EARLY AND LATE ACTIVITY IN SOMATOSENSORY CORTEX REFLECTS CHANGES IN BODILY SELF-CONSCIOUSNESS: AN EVOKED POTENTIAL STUDY
}

\author{
J. E. ASPELL, ${ }^{a *}$ E. PALLUEL ${ }^{a}$ AND O. BLANKE ${ }^{a, b}$ \\ a Laboratory of Cognitive Neuroscience, Ecole Polytechnique \\ Fédérale de Lausanne (EPFL), Lausanne, Switzerland \\ ${ }^{\mathrm{b}}$ Department of Neurology, University Hospital, Geneva, Switzerland
}

\begin{abstract}
How can we investigate the brain mechanisms underlying self-consciousness? Recent behavioural studies on multisensory bodily perception have shown that multisensory conflicts can alter bodily self-consciousness such as in the "full body illusion" (FBI) in which changes in self-identification with a virtual body and tactile perception are induced. Here we investigated whether experimental changes in self-identification during the FBI are accompanied by activity changes in somatosensory cortex by recording somatosensory-evoked potentials (SEPs). To modulate self-identification, participants were filmed by a video camera from behind while their backs were stroked, either synchronously (illusion condition) or asynchronously (control condition) with respect to the stroking seen on their virtual body. Tibial nerve SEPs were recorded during the $\mathrm{FBI}$ and analysed using evoked potential (EP) mapping. Tactile mislocalisation was measured using the crossmodal congruency task. SEP mapping revealed five sequential periods of brain activation during the $\mathrm{FBI}$, of which two differed between the illusion condition and the control condition. Activation at $\mathbf{3 0}-\mathbf{5 0} \mathrm{ms}$ (corresponding to the P40 component) in primary somatosensory cortex was stronger in the illusion condition. A later activation at $\sim 110-200 \mathrm{~ms}$, likely originating in higher-tier somatosensory regions in parietal cortex, was stronger and lasted longer in the control condition. These data show that changes in bodily self-consciousness modulate activity in primary and higher-tier somatosensory cortex at two distinct processing steps. We argue that early modulations of primary somatosensory cortex may be a consequence of (1) multisensory integration of synchronous vs. asynchronous visuo-tactile stimuli and/or (2) differences in spatial attention (to near or far space) between the conditions. The later activation in higher-tier parietal cortex (and potentially other regions in
\end{abstract}

\footnotetext{
*Corresponding author. Address: Department of Psychology, Faculty of Science and Technology, Anglia Ruskin University, Cambridge, CB1 1PT, United Kingdom. Tel: +44-1223-363271x2258.

E-mail addresses: jane.aspell@anglia.ac.uk, jane.aspell@epfl.ch (J.E. Aspell).

Abbreviations: ANOVA, analyses of variance; CCE, crossmodal congruency effects; EEG, electroencephalography; EP, evoked potential; FBI, full body illusion; GFP, global field power; HMD, head mounted display; LED, light emitting diode; RHI, rubber hand illusion; RT, reaction time; SEP, somatosensory-evoked potential; TPJ, temporoparietal junction.
}

temporo-parietal and frontal cortex) likely reflects the detection of visuo-tactile conflicts in the asynchronous condition. (C) 2012 IBRO. Published by Elsevier Ltd. All rights reserved.

Key words: body perception, self-consciousness, somatosensory, electroencephalography, multisensory integration.

\section{INTRODUCTION}

Body ownership (the sense that my body belongs to me) is a crucial feature of bodily self-consciousness, the nonconceptual and pre-reflective representation of bodyrelated information (Gallagher, 2005; Jeannerod, 2007; Blanke and Metzinger, 2009). Recent work shows that this apparently deeply rooted aspect of human experience is, to some degree, modifiable. Thus, visuo-tactile conflicts can induce measurable changes in self-attribution of a fake hand in the rubber hand illusion (Botvinick and Cohen, 1998), and in self-identification with a whole virtual body in the full body illusion (FBI) (Lenggenhager et al., 2007, 2009); see also (Ehrsson, 2007). Changes in tactile perception have also been found to accompany changes in bodily self-consciousness: a recent study demonstrated the modulation of touch by measuring crossmodal congruency effects (CCEs) - derived from repeated reaction time (RT) and accuracy measurements - during the FBI (Aspell et al., 2009). This study demonstrated that the modulation of self-identification was also reflected in differences in CCE magnitude, providing strong evidence for the mislocalisation of touch towards a virtual body during the illusion. A similar CCE demonstration of tactile mislocalisation was found when participants viewed a rubber hand (Pavani et al., 2000; Heed et al., 2010). In the present study we investigated whether these changes in self-identification and tactile perception during the $\mathrm{FBI}$ are accompanied by changes in somatosensory cortex.

Not much is known about which brain mechanisms underlie the induced changes in self-identification with a virtual body or avatar, but three recent FBI studies have begun to answer this question. The first, using frequency analysis and high resolution electroencephalography (EEG), showed that primary somatosensory cortex and medial prefrontal cortex reflect changes in self-identification and self-location during the FBI (Lenggenhager et al., 2011) and the second, an functional magnetic resonance imaging (fMRI) study, revealed that activation of the right temporo-parietal junction is modulated by 
changes in self-location (lonta et al., 2011). Another fMRI study of a similar FBI, the "body swap" illusion found that activation in the left intraparietal and bilateral ventral premotor cortices (and left putamen) was greater in the illusion condition than in the asynchronous control condition (Petkova Valeria et al., 2011). Differences between the findings of these FBI studies are likely due to variations in the experimental setup (viewing a mannequin vs. an animated avatar; first person vs. third person perspective, etc.) and in the methods used to measure brain activity. Related neuroimaging studies of selfattribution of a fake hand during the rubber hand illusion $(\mathrm{RHI})$ implicated a wide network of similar brain regions including the intraparietal cortex, primary somatosensory cortex, the right temporo-parietal junction, the ventral premotor cortex and the right insular lobe (Ehrsson et al., 2004, 2005, 2007; Tsakiris et al., 2007, 2008).

Parietal cortex has also been implicated in self-attribution by studies employing somatosensory-evoked potentials (SEPs) and frequency analysis. For example, (Kanayama et al., 2007, 2009) reported that gamma band oscillations $(40-50 \mathrm{~Hz})$ over parietal scalp regions varied according to RHI strength. One SEP study (Press et al., 2008) showed enhancement of a late somatosensory SEP component (evoked by hand tapping) after a period of synchronous stroking of a rubber hand, likely reflecting activation of higher-tier somatosensory regions in parietal cortex (and/or premotor cortex), whereas a different illusion paradigm using SEPs implicated primary somatosensory cortex (Dieguez et al., 2009), based on the observation that the earliest cortical SEP component after median nerve stimulation (N20 component) was enhanced. In summary, these data, using a variety of tasks and neuroimaging methods support an implication of parietal cortex in self-identification, but do not enable us to distinguish between activity changes in primary somatosensory cortex vs. higher-tier regions in parietal cortex.

Here, to specifically investigate the role of somatosensory cortex, we investigated the timing and location of brain activity during a state of altered bodily self-consciousness by recording SEPs to stimulation of the tibial nerve of the lower leg during the FBI. SEP components to electrical stimulation (most commonly of the median nerve) have been classified as short and long latency (Allison et al., 1989a,b, 1991). Short latency components are found at $40 \mathrm{~ms}$ or less, and are generated in contralateral area $3 \mathrm{~b}$ of SI. Long-latency (>40 ms) components are thought to be generated by several areas, including, in addition to area $3 \mathrm{~b}$, areas 1 and 2 , secondary somatosensory cortex (SII), and primary motor cortex (area 4). There have been fewer studies of SEPs to lower limb (tibial nerve) stimulation than to upper limb (median nerve) stimulation but it is known that the latency and topography of tibial nerve SEP components differs from median nerve SEPs, because of longer signal conduction times (given the greater ankle to brain than wrist to brain distance) and because of the different locations of leg and arm representation in primary somatosensory cortex (Jones and Small, 1978; Kany and Treede, 1997). Thus, for tibial stimulation the P40 component has generally been considered to be the first cortical potential (short-latency component) and is generally recorded $20-30 \mathrm{~ms}$ later than the first cortical potential - the N20 - to median nerve stimulation in the same participant (Kany and Treede, 1997). Like the N20, the P40 is thought to be generated in area 3b of SI (Kakigi et al., 1995), but is characterised by 'paradoxical lateralization', i.e. tibial nerve SEP amplitude is greater in the ipsilateral rather than contralateral hemisphere (Cruse et al., 1982).

For the present study, we adapted the recent SEP approach used by Dieguez et al. (2009) to the FBI setup. In order to record brain activity relevant to illusory selfidentification with a virtual body we recorded SEPs in response to tibial nerve stimulation because full body representations depend on somatosensory processing from the lower limbs and because we have previously shown that self-identification and associated tactile changes (measured by the CCE) are modulated by somatosensory (proprioceptive) signals delivered to the legs but not to the wrists (Schwabe and Blanke, 2008; Palluel et al., 2011, 2012). We predicted that early activity in primary somatosensory cortex (40 ms after tibial nerve stimulation (Kakigi et al., 1982)) would reflect changes in self-identification during the $\mathrm{FBI}$ and, according to (Dieguez et al., 2009), that it would be enhanced during the illusion condition. We also measured the CCE during the illusion, in the same blocks as the tibial nerve stimulation, in order to test whether a change in tactile mislocalisation would also occur with the current setup, as found previously for the FBI (Aspell et al., 2009). Furthermore, this should enable us to directly compare - in the same participants and the same study - this behavioural measure of a change in tactile processing with an electrophysiological (SEP) measure.

\section{EXPERIMENTAL PROCEDURES}

\section{Participants}

A total of 18 right-handed healthy volunteers took part. All participants gave written informed consent and were compensated for their participation. The study protocol was approved by the local ethics research committee - La Commission d'éthique de la recherche Clinique de la Faculté de Biologie et de Médecine at the University of Lausanne, Switzerland and was performed in accordance with the ethical standards laid down in the Declaration of Helsinki. Data from six participants had to be discarded because they did not show an identifiable SEP (inter-participant SEP amplitude variability is known to be large (Ferri et al., 1996; Gardill and Hielscher, 2001; van de Wassenberg et al., 2008)).

\section{Materials}

The FBI was combined with a behavioural task that allowed us to assess changes in bodily self-consciousness during the illusion (CCE). For the CCE task we employed four 'light-vibration' devices, each consisting of a single bright light emitting diode (LED) paired with a small-vibrating motor (for full details see (Aspell et al., 2009)). The devices were attached to the skin using surgical tape. The two 'upper' devices were positioned at the inner edges of the shoulder blades and the two 'lower' devices $9 \mathrm{~cm}$ below (Fig. 1). The experiments were performed in an electrically-shielded Faraday cage. Participants were seated on 
A

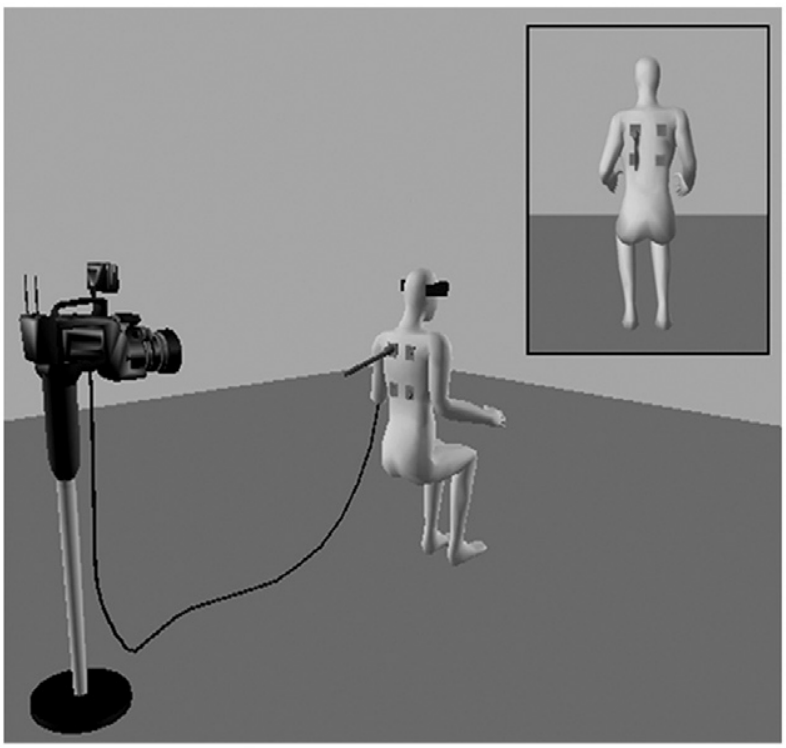

B

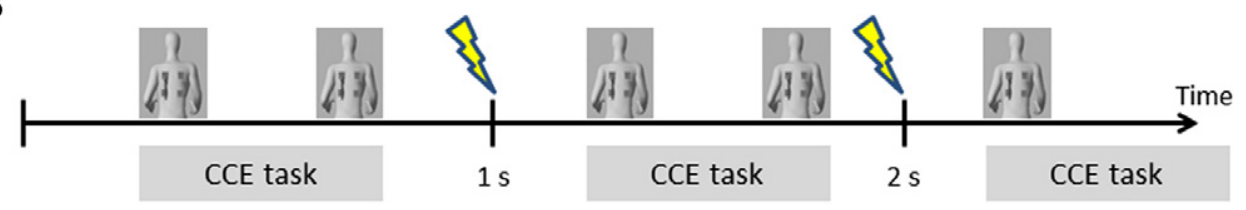

Stroking

Tibial nerve stimulation

CCE task The CCE consisted of a visual stimulus ( $33 \mathrm{~ms})$, SOA ( $233 \mathrm{~ms}$ ) and a vibratory stimulus (100 ms)

Fig. 1. (A) Experimental set-up. Participant was sat $2 \mathrm{~m}$ in front of a video camera. Four light-vibration devices were fixed to the participant's back, the upper two at the inner edges of the shoulder blades and the lower two $9 \mathrm{~cm}$ below. Figure shows synchronous stroking condition. The small inset window represents what the participant viewed via the head mounted device. (B) Schematic representation of a trial with the timing of the stimuli. Note that there was no match between the timing of the CCE - i.e. visual stimulus, stimulus onset asynchrony (SOA) and tactile stimulus - and the tibial nerve stimulation.

a stool with their backs facing a video camera located $2 \mathrm{~m}$ behind. The video was projected in real time (except for in asynchronous blocks, see below) onto a head-mounted display (HMD) enabling participants to view the video of their bodies. During both blocks - synchronous and asynchronous - the backs (the area spanning the shoulders to waist) of participants were irregularly stroked - about twice per second - by the experimenter with a long wooden stick, and participants could view the stroking via the HMD. The stroking began one minute before the first vibro-tactile stimulus was presented, and continued throughout the entire block. In asynchronous blocks a delay of $400 \mathrm{~ms}$ was introduced (using a video delaying device) so that 'seen stroking' and 'felt stroking' did not correspond.

Each CCE trial consisted of a light (LED) flash followed by a vibro-tactile stimulus. The active LED and active-vibrating motor were varied randomly and independently from trial to trial. Each trial began with a light flash of $33 \mathrm{~ms}$ duration and the vibro-tactile stimulus was presented $233 \mathrm{~ms}$ after the light onset, and for a duration of $100 \mathrm{~ms}$. After participants had responded with a button press there was a $1 \mathrm{~s}$ pause before the succeeding trial commenced. There were 100 trials per block, 25 per combination (same side, congruent elevation, same side incongruent elevation, different side congruent elevation, different side, incongruent elevation).

To stimulate the tibial nerve we attached two skin electrodes to the inside ankle of the right leg and used a Grass S48 stimulator to generate electrical pulses. During each block the nerve was stimulated 400 times at a duration of $0.2 \mathrm{~ms}$, a frequency of $1 \mathrm{~Hz}$ and at an intensity just below motor threshold (Hume and Cant, 1978). No participant reported pain or discomfort with this level of stimulation. Note that there was no attempt to match the timing of the CCE trials and the tibial nerve stimulation (the timing of successive CCE trials depended on the response times to each trial, thus their onset times were not perfectly regular throughout the blocks). In this way we excluded that visual and tactile stimulation onset related to CCEs confounded electrical SEP stimulation onset.

\section{Procedure}

Participants were instructed to keep their eyes open during testing blocks and fixate a location in the middle of their backs, as viewed via the HMD. The tibial nerve stimulations began at the 
Table 1. Self-identification questionnaire

\begin{tabular}{ll}
\hline During the experiment there were times when: \\
\hline 1 & $\begin{array}{l}\text { It seemed as if I was feeling the touch of the stick in the } \\
\text { location where I saw the virtual body being touched }\end{array}$ \\
2 & $\begin{array}{l}\text { It seemed as though the touch I felt was caused by the stick } \\
\text { touching the virtual body }\end{array}$ \\
3 & I felt as if the virtual body was my body \\
4 & It felt as if my (real) body was drifting towards the front \\
5 & $\begin{array}{l}\text { It seemed as if I might have more than one body } \\
6\end{array} \begin{array}{l}\text { It seemed as if the touch I was feeling came from } \\
\text { somewhere between my own body and the virtual body }\end{array}$ \\
7 & $\begin{array}{l}\text { It appeared (visually) as if the virtual body was drifting } \\
\text { backwards (towards my body) }\end{array}$ \\
8 & It seemed as though I was in two places at the same time \\
\hline
\end{tabular}

start of the block - when the stroking began - and continued throughout the duration of the block. For the first minute of each block no vibro-tactile or LED stimuli were presented and participants were instructed to sit still, watching the stroking, and wait for the first stimulus. For each CCE trial, participants had to signal with their right hand, by pressing one of two buttons as fast as possible, whether they felt a vibration at the top (an upper device) or at the bottom (a lower device) of their backs (regardless of side), while trying to ignore the light flashes. These responses enabled us to measure RTs and accuracies. At the end of the block (of duration $\sim 9 \mathrm{~min}$ ) self-identification with the seen body and other phenomenological aspects were assessed by means of a questionnaire (see Table 1) adapted from (Lenggenhager et al., 2007). Participants took a short break before the second block. All participants completed a training session (with the body visible, no stroking and no tibial nerve stimulations) prior to the experimental blocks. The order of blocks (synchronous/asynchronous) was counterbalanced across participants. Note that we were not able to measure the drift in self-location towards the virtual body (measured in previous studies) because participants were sitting and their movements were constrained by the attachment of the EEG and stimulation leads.

\section{EEG recordings - acquisition and preprocessing}

Continuous EEG (BioSemi, The Netherlands) was recorded at a sample frequency of $8.192 \mathrm{~Hz}$ from 32 scalp electrodes that were evenly spaced according to the 10-20 EEG system. Electrodes included conventional midline sites $\mathrm{Fz}, \mathrm{Cz}, \mathrm{Pz}, \mathrm{Oz}$ and sites over the left and right hemispheres. Electrooculogram (EOG) was also recorded to control for eye movements. EEG epochs were calculated from $100 \mathrm{~ms}$ before to $600 \mathrm{~ms}$ after the onset of the tibial nerve stimulation. A baseline correction was calculated from 100 to $20 \mathrm{~ms}$ before stimulus onset. An automated artefact rejection threshold of $\pm 50 \mu \mathrm{V}$ was used based on EEG and EOG channels and was adjusted for each individual participant (Mercier et al., 2009). All accepted trials were also visually inspected to reject epochs with transient noise such as eye blinks and muscle artefacts. A $50 \mathrm{~Hz}$ notch filter was applied to reduce persistent (e.g. electrical) noise. SEPs were bandpass-filtered $(1-40 \mathrm{~Hz})$

\section{SEP analysis}

Grand average SEPs across participants were calculated with evoked potentials (EPs) normalised to the global field power (GFP) (the spatial standard deviation of the scalp electrical field at a given moment (Mercier et al., 2009)). We performed evoked potential (EP) mapping (Lehmann et al., 1987) to investigate differences in brain activity across the two conditions. In the single traces we identified classical early SEP components after electrical tibial nerve stimulation (P40, N50 and P60) for each individual participant according to polarity and latency in the grand average SEP.

EP topographical analysis was based on the examination of spatial variations of the scalp voltage distribution over time and between tasks. In brief, the EP topographical mapping approach consists of two main analysis steps (Michel et al., 2001; Blanke et al., 2005; Arzy et al., 2006; Murray et al., 2008; Mercier et al., 2009) and searches for time periods of stable map topography within and across experimental conditions. In the first analysis step, EP microstate segments (EP topographies or EP maps) were defined by using a spatial clustering algorithm (Tibshirani and Walther, 2005). The cluster analysis step is dependent upon the quantification of the global explained variance (GEV) which corresponds to the goodness of fit of a template map during a certain time period and the instantaneous strength of the electrical field (GFP; (Lehmann and Skrandies, 1980; Murray et al., 2008). This analysis identifies the dominant map topographies on the scalp in the group-averaged SEPs across both experimental conditions over time. In the second analysis step we performed statistical analysis and verified the presence of a given EP map as identified in the group-averaged data in the EPs of the individual participants. This is done by means of a fitting procedure based on the spatial correlation between template maps obtained from the group-averaged EPs data and the individual participants' data (Lopez et al., 2011). Thus, the dominant EP maps, as identified by the segmentation procedure in the group-averaged data (analysis step 1), were fitted to the EPs of each individual participant (analysis step 2). The fitting procedure is based on the spatial correlation between template EP maps obtained from the group-averaged EPs data and the individual participant data and identical to that done in our previous EP mapping work (Blanke et al., 2005; Arzy et al., 2006; Mercier et al., 2009).

\section{Analysis of behavioural data}

Trials with incorrect responses and trials in which participants failed to respond within $1500 \mathrm{~ms}$ were discarded from the RT analysis (following the method of (Spence et al., 2004)). The mean RTs were analysed using two-tailed repeated measures analyses of variance (ANOVA) with three factors: synchrony (synchronous/asynchronous), congruency (congruent/incongruent) and side (same/different). In order to analyse the illusion strength as determined by the questionnaire ratings we compared the ratings in the illusion questions (Questions 1-3) with the ratings of the control questions (Questions 4-7, questions that were not related to the illusion and instead controlled for suggestibility) in the two experimental conditions (score between -3 and 3). For statistical analysis we used an ANOVA with the factors-stroking type (synchronous/asynchronous), and question type (illusion/control); see (Slater et al., 2008; Petkova and Ehrsson, 2009). The significance (alpha) level used was 0.05 .

\section{RESULTS}

\section{Behavioural results}

For the questionnaire data, the ANOVA revealed a significant main effect of question type, as ratings for the illusion questions were significantly greater than for the control questions $\left(F_{1,11}=5.78 ; p=0.035\right)$. It further revealed a main effect of synchrony, as synchronous stroking was associated with greater rating scores $\left(F_{1,11}=3.84 ; p=0.026\right)$. Crucially we found an interaction of stroking type $\times$ question type $\left(F_{1,11}=10.3\right.$; $p=0.008)$ revealing the selective manipulation of the illusion questions by the synchrony of stroking: planned comparisons showed - as predicted - that self-identification was greater in the synchronous condition than in the 
asynchronous condition for the illusion questions ( $p=$ 0.003 ) but not for the control questions ( $p=0.526)$, see Fig. 2. These data confirm questionnaire data from previous studies (Ehrsson, 2007; Lenggenhager et al., 2007; Aspell et al., 2009); revealing greater self-identification during synchronous than asynchronous stroking.

Fig. 3 plots the size of the CCE (RT in incongruent trials minus RT in congruent trials) for the synchronous and asynchronous conditions. As expected, in both conditions the CCE was larger when the light appeared on the same side as the tactile stimulus, compared to when it appeared on the different side. Statistical analysis revealed a significant main effect of congruency $\left(F_{1,11}=15.6 ; p=0.002\right)$ and a significant interaction between side and congruency $\left(F_{1,11}=19.5 ; p=0.001\right)$. Although CCEs were numerically larger during synchronous than asynchronous stroking (Fig. 3), they did not differ significantly between these conditions. No other main effects or interactions reached significance.

The behavioural data from the present study is consistent with previous studies (Lenggenhager et al., 2007; Aspell et al., 2009) of the $\mathrm{FBI}$ : self-identification was greater in the synchronous condition than in the control condition. The failure to find a significant effect of synchrony on the CCE may be due to the fact that the

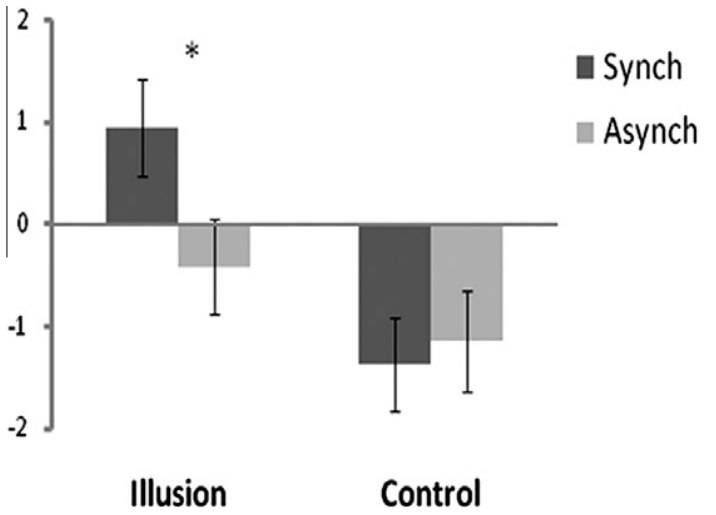

Fig. 2. Questionnaire results. Error bars show standard error of the mean. present experimental conditions were arguably less optimal for the visual capture of touch that usually occurs during the illusion: participants were positioned within a Faraday cage, close to its corner walls and this may have lessened the illusion that the virtual body was located $2 \mathrm{~m}$ in front of them (our previous studies used a large room with the facing wall at least $2 \mathrm{~m}$ distant). It is also possible that the tibial nerve stimulation which occurred during the CCE task may have distracted participants and/or somehow interfered with the tactile processing of the vibro-tactile CCE stimuli.

\section{EEG results}

EP analysis revealed three classic early SEP components peaking at P40, N50 and P60 (see Fig. 4). These were maximal for both conditions at electrodes CP2, FC2, Fz and $\mathrm{Cz}$. No clear components could be identified after $100 \mathrm{~ms}$. In a single trace analysis we selected three time windows: $35-45,45-55$ and 55-65 ms and ran ANOVAs for each main electrode (Fz, Cz, Pz, FC1, C3, CP1, FC2, C4 and CP2) with two factors: synchrony and window. There were no significant differences between the conditions.

Segmentation of the group-averaged data revealed five sequential segments of brain activation (up to $200 \mathrm{~ms}$ ) following the electrical stimulation in both conditions. Yet, only two of these maps (maps 2 and 5) differed between the experimental conditions, whereas the remaining three maps (maps 1,3 , and 4) were present in both conditions and did not differ between them. Map 2 appeared from 38 to $50 \mathrm{~ms}$ after stimulus onset (in light-grey, see Fig. $5 a$ and b) and predominated in the synchronous condition. Statistical analysis (two-tailed $t$-tests) showed that map 2 had a stronger amplitude (reflected in a higher mean GFP $\left(F_{1,11}=5.03\right.$; $p=0.046)$ in the synchronous $(0.35 \pm 0.04 \mu \mathrm{V})$ as compared to the asynchronous condition $(0.27 \pm 0.06 \mu \mathrm{V})$. No difference in the duration of map 2 between the conditions was found ( $p=0.19)$.

The later map 5 (black, see Fig. $5 a, c)$ appeared from $\sim 115$ to $200 \mathrm{~ms}$ and was found to predominate in the

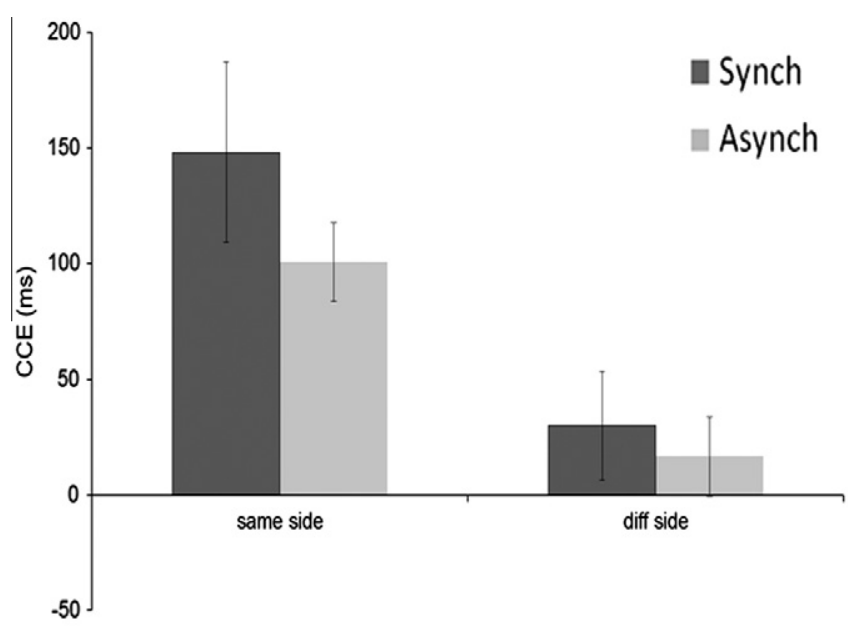

Fig. 3. Mean crossmodal congruency effects (CCEs) in reaction time (RT) in milliseconds (RT in incongruent trials minus RT in congruent trials). 

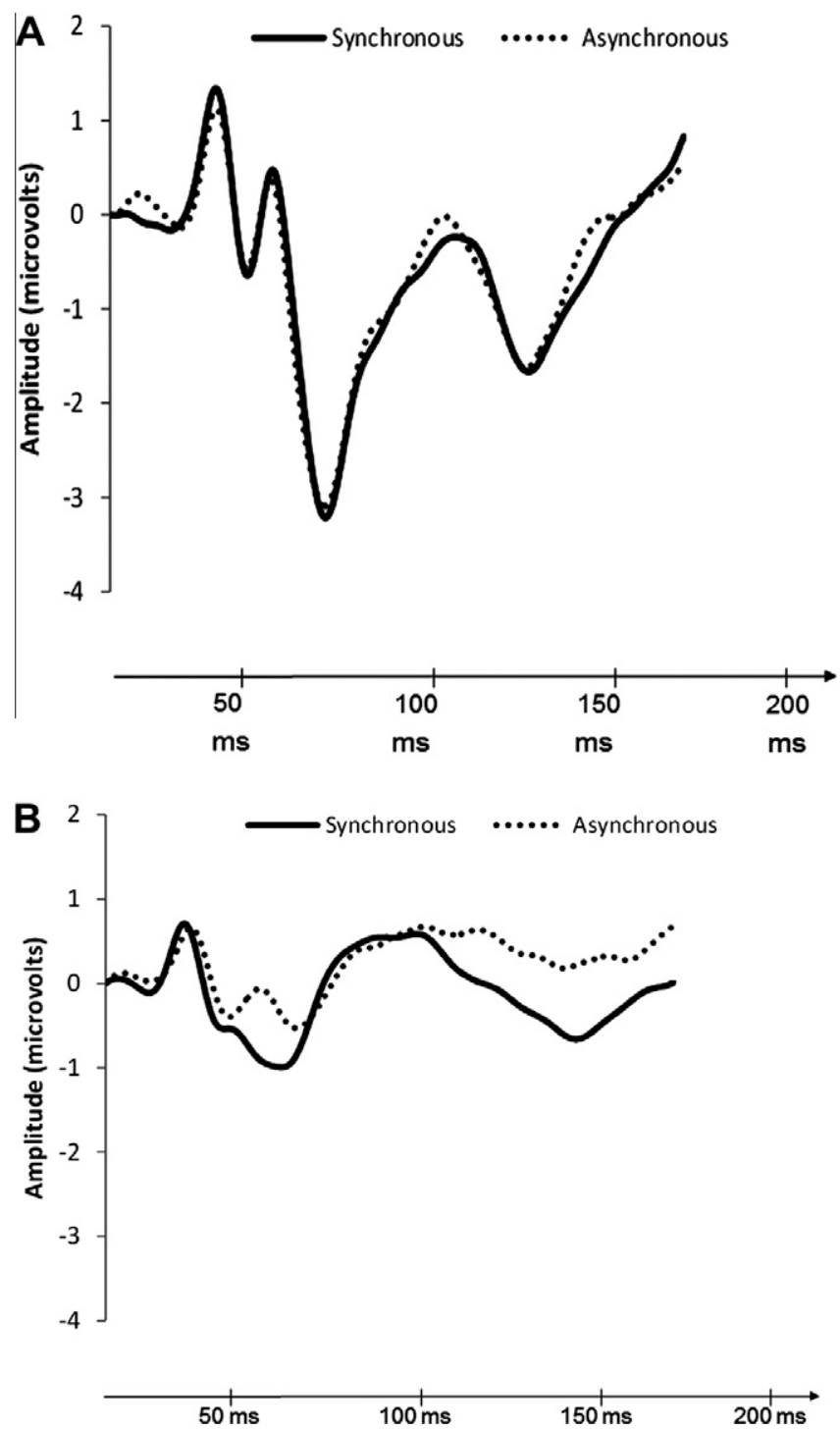

Fig. 4. Superimposed average waveforms in the two conditions (A) electrode $\mathrm{Cz}$ for one participant (B) electrode $\mathrm{C} 4$ for one participant.

asynchronous condition. The mean GFP was higher in the asynchronous condition $\left(F_{1,11}=5.09 ; p=0.045\right.$; $0.31 \pm 0.04 \mu \mathrm{V})$ as compared to the synchronous condition $(0.38 \pm 0.04 \mu \mathrm{V})$. Furthermore, the map duration was significantly $\left(F_{1,11}=5.88 ; p=0.034\right)$ longer in the asynchronous condition $(62.7 \pm 24.7 \mathrm{~ms})$ as compared to the synchronous condition ( $41.47 \pm 5.9 \mathrm{~ms})$. No significant topographical effects were found during other periods.

The scalp topography of maps 2 and 5 is shown in Fig. $5 \mathrm{~b}$ and $\mathrm{c}$. The electrode with maximal amplitude for map 2 was found at electrode Pz and for map 5 at electrode F4. Due to the low number of electrodes no linear inverse solution was applied to the data (as done for example in Schwabe et al., 2009; Arzy et al., 2006).

\section{DISCUSSION}

The present study shows that activity modulations in somatosensory cortex, as measured with SEPs, accompany changes in bodily self-consciousness induced by the FBI. Even under the constraints of the present setup - co-application of a mild electrical shock to the leg and co-recording of EEG - we found comparable behavioural effects with respect to previous data obtained in less constraining conditions (Ehrsson, 2007; Lenggenhager et al., 2007; Aspell et al., 2009). Our main interest here was the investigation of processing in somatosensory cortex during the FBI by measuring SEPS to tibial nerve stimulation. We show that changes in bodily self-consciousness induced by the FBI (Lenggenhager et al., 2007) are associated with modulations of SEPs during two distinct processing steps. Brain activity at around the time of the first parietal component of tibial nerve SEPs (P40) was found to be enhanced during the illusion. This finding as well as the associated SEP scalp map which has a maximum at the central electrode Pz suggests that the FBI modulates activity in the S1 leg representation. A later response - at 110-200 ms - had greater amplitude and longer duration during the control 
A
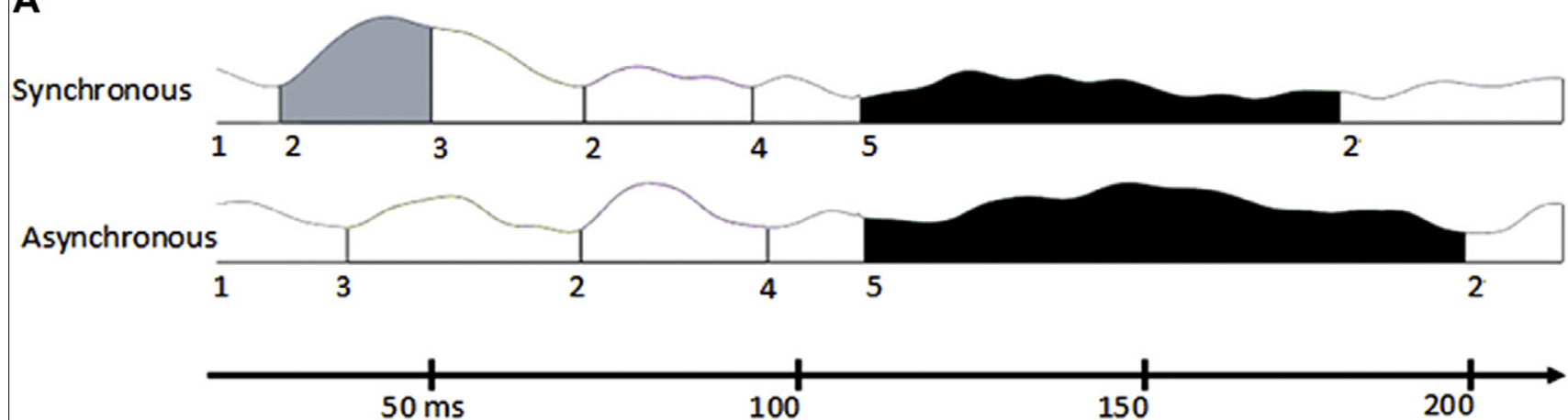

B
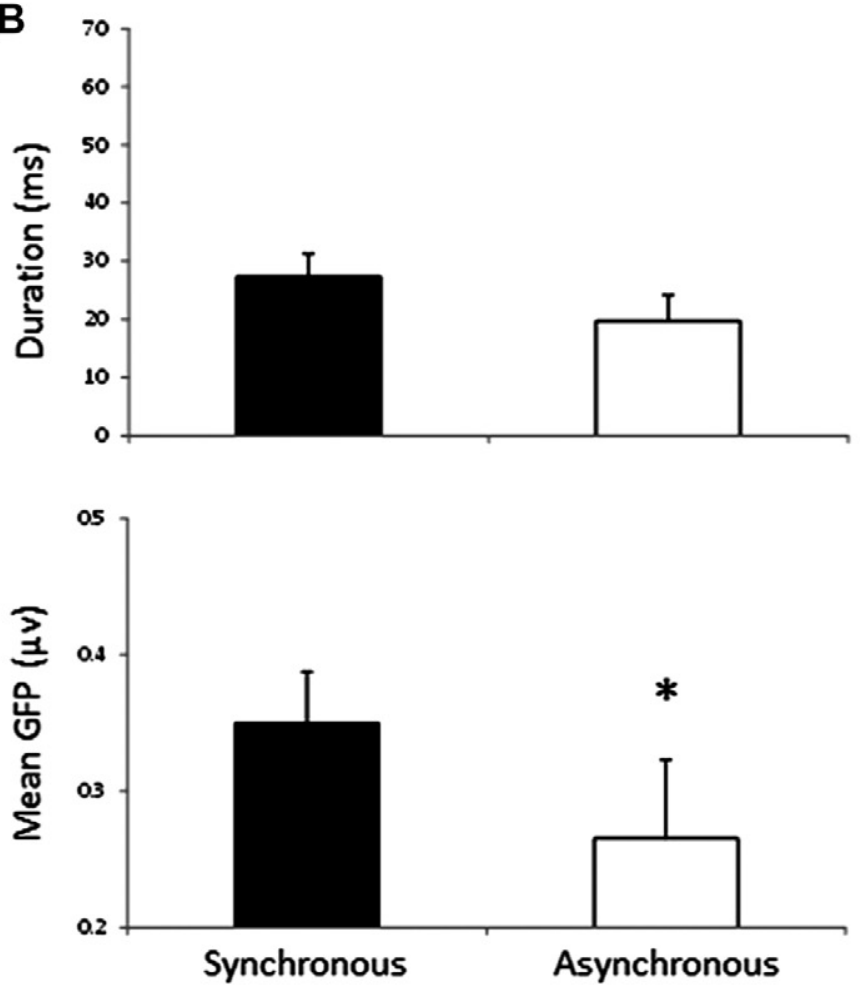

C

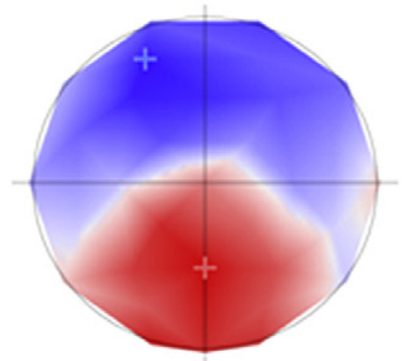

Map 2
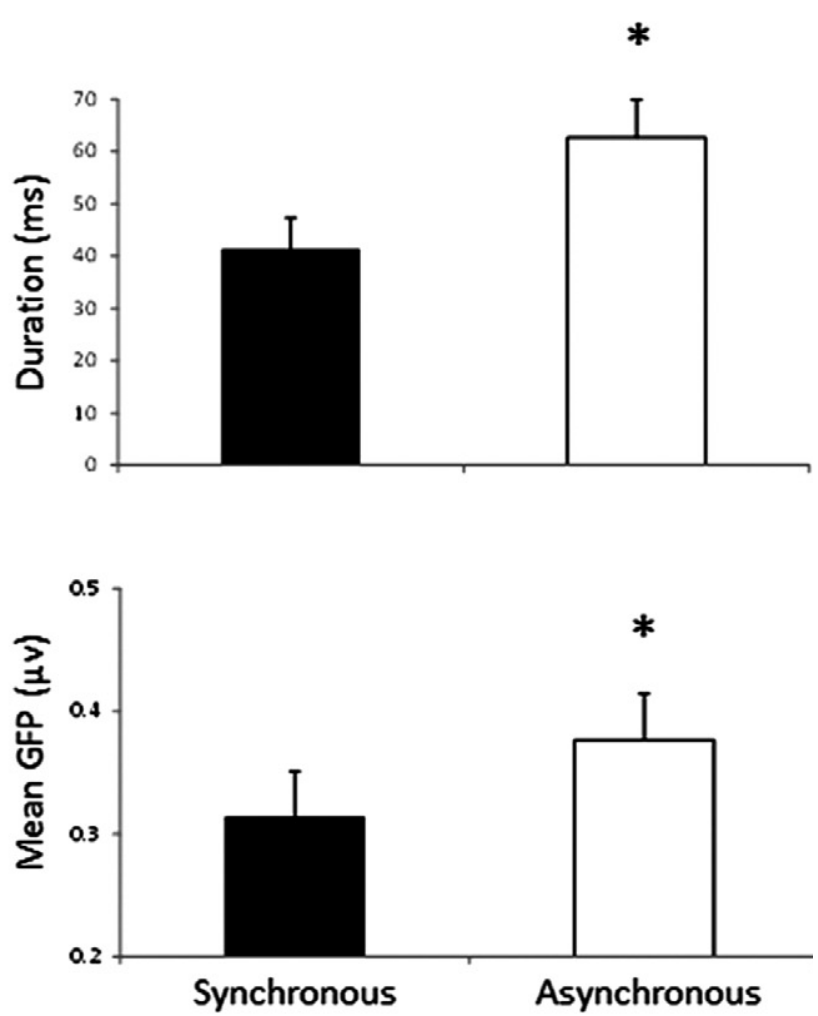

Map 5

Fig. 5. EEG results: (a) segments of stable map topography in the two conditions (asynchronous, synchronous) under the global field power (GFP, in microvolts) curve from 0 to 200 ms after the stimulation (top). (b) Plots show map duration (top) and mean GFP (bottom) for maps 2 (left) and 5 (right). Error bars show standard error of the mean. (c) Map topographies for maps 2 and 5 are shown (middle).

condition and was found at a more frontal scalp region, peaking at electrode F4. These differences in timing and dependence on illusion strength reveal the involvement of parietal mechanisms in the $\mathrm{FBI}$ and distinct brain mechanisms at these two processing steps.
Regarding the neural generators of these SEP responses, previous studies have localised SEP components at similar latencies. Several studies (Seyal et al., 1983; Chiappa, 1997; Miura et al., 2003) have shown a degree of inter-individual variability in the topography of 
the P40 (greater than that of the N20 component to median nerve stimulation) possibly because of the known variation in the location of the leg representation in SI (Kakigi et al., 1995). In the present study we did not find an effect of the different synchrony conditions in single trace analysis and this may be related to this variability in leg representation and also to the co-application of the CCE vibro-tactile stimulus (in addition to the tactile stroking) which could have interfered with the SEPs recorded at single electrodes. The average-referenced multielectrode based topographical SEP map changes may be more robust (see also (Murray et al., 2008; Lascano et al., 2009; Michel and Murray, 2012)), at least in the present experimental setup. Topographic analyses can be interpreted with respect to neurophysiology since differences in scalp potential topography directly reveal changes in the configuration of neural generators. There are also statistical reasons for using the entire montage rather than single selected channels since the former takes fuller advantage of all the data and so arguably increases the rigour of the statistical analyses.

Although a few studies (Desmedt and Bourguet, 1985; Caselli, 1993; Casey et al., 1994) have reported that some tibial nerve SEP components are generated in frontal and parietal areas, others have failed to find generators here, e.g. Kakigi et al. (1995). The debate about whether the P40 is generated exclusively in SI or also has additional generators in other areas is ongoing (Baumgärtner et al., 1998). SEP components to tibial nerve stimulation around the time period of the later response at $110-200 \mathrm{~ms}$ are reported to be generated in or near to SII and they may also have generators in posterior parietal and frontal cortex (Mountcastle, 1984; Allison et al., 1989a, 1991; Desmedt and Tomberg, 1989; Forss et al., 1994, 1996; Kakigi et al., 1995; Kany and Treede, 1997). Accordingly, we argue that the modulation of brain activity associated with the later map 5 reflects activation of a larger network including mainly posterior parietal, temporo-parietal, and frontal regions, as compared to the earlier activation (map 2) that most likely reflects mainly $\mathrm{S} 1$ activation.

Modulations of the earliest somatosensory components to electro-tactile stimulation (e.g. the N20 to median nerve stimulation and corresponding P40 to tibial nerve stimulation) are most commonly reported as being modulated by limb movements/motor interference (Abbruzzese et al., 1980; Rossini et al., 1996; Valeriani et al., 1998; Asanuma et al., 2003; Gobbelé et al., 2003; Kida et al., 2004; Legon and Staines, 2006). What accounts for our finding of an enhancement of SEP amplitude during the illusion for the earlier response and the reverse effect for the later response? We discuss the following three non-exclusive brain mechanisms: (1) multisensory integration effects, (2) attentional effects and (3) 'functional deafferentation' associated with disownership of one's physical body during the FBI.

\section{Multisensory integration effects}

It is possible that the SEP differences between the synchronous and asynchronous conditions are due to the modulation of unisensory (tactile) processing in SI by the multisensory (visuo-tactile) integration of the seen and felt stroking (see also (Sathian and Stilla, 2010). In the synchronous illusion condition the visual and tactile stimuli are characterised as providing information about a single event and thus they are integrated. Our findings are thus compatible with a large body of evidence that visuo-tactile integration may enhance brain activity measured by EPs in early, modality specific cortices, including somatosensory cortex (see, e.g. (Eimer et al., 2001; Taylor-Clarke et al., 2002) and for a review see (Eimer and Driver, 2001)). Our finding of a stronger early activation in the synchronous condition may therefore be due to such early, bottom-up multisensory enhancement effects (Macaluso and Driver, 2005; Macaluso and Maravita, 2010) resulting from the integration of congruent visual and tactile (stroking) cues during the FBI.

In contrast, in the asynchronous control condition the stimuli are more likely processed as if they represent two separate events or a multisensory mismatch and there should in this case be a reduction in multisensory integration. A number of fMRI studies have reported activation of the temporo-parietal junction (TPJ) when multisensory predictions are violated (Downar et al., 2000; Corbetta and Shulman, 2002). The stronger activations for the later map in the asynchronous condition might also be related to mechanisms of greater multisensory bodily conflict (Tsakiris et al., 2007; Lenggenhager et al., 2011). Thus, in the asynchronous and synchronous body conditions participants were exposed to spatial incongruency between the seen virtual body and the felt body (participant's body). However, there was a second conflict only in the asynchronous condition - a spatio-temporal incongruency concerning the relative location of the seen and felt touch on the back. The stronger activation during the later time period (from 110-200 ms) for the asynchronous condition may therefore reflect the detection of incongruency or conflict in the asynchronous condition by multisensory cortical areas which integrate the visual and tactile stroking cues and which can modulate somatosensory cortex via back projections (Taylor-Clarke et al., 2002; Macaluso and Driver, 2005; Macaluso and Maravita, 2010). Finally, the later SEP modulation may also be related to error/mismatch detection during the processing of non-matching (asynchronous) visual and tactile inputs. EP correlates of error/mismatch detection are typically fronto-central components and are found at a similar duration to that of map 2 yet they usually require the presence of single deviant stimuli (Näätänen, 1975; Näätänen et al., 1978; Folstein and Van Petten, 2008). Such components are usually found in the context of cognitive, e.g. oddball, tasks, and the present setup could invoke related cognitive processes since there is a strong expectation of seeing the touch in the same place on the virtual body as where the touch is felt. The asynchronous condition violates this expectation.

It is worth comparing the present data with findings from two recent FBI fMRI studies (Ionta et al., 2011; Petkova Valeria et al., 2011) which also measured brain activation during synchronous and asynchronous stroking conditions. In one of these FBI studies (Ionta et al., 2011), 
the TPJ region was shown to be modulated by visuotactile synchrony which may be related to the finding (mentioned above) that TPJ activation can be modulated by the violation of multisensory predictions (Downar et al., 2000; Corbetta and Shulman, 2002). The fMRI study used a version of the $\mathrm{FBI}$ very similar to that in the present study (but in supine-positioned participants) and induced changes in self-location towards a virtual body viewed as from a third person perspective (a sort of disembodiment). The second fMRI study (Petkova Valeria et al., 2011) used the "body swap illusion" in which participants feel ownership for a mannequin's body viewed from the first person perspective (thus no changes in self-location occur) and it is notable that different brain regions - ventral premotor and intraparietal cortex (but not the TPJ) were modulated by synchrony during this illusion. We note that all three of these brain regions are known to be important areas for multisensory integration (see e.g. (Downar et al., 2000; Bremmer et al., 2001)).

As discussed earlier, a recent EEG study of the $\mathrm{FBI}$ using high-density frequency analysis (Lenggenhager et al., 2011) found that activation in somatosensory cortex (as well as other central and frontal brain areas) differed during synchronous and asynchronous stroking. This EEG study examined differences in online continuous EEG during the illusion and control conditions and demonstrated that brain activity in bilateral medial sensorimotor (including somatosensory cortex) and premotor cortices showed significantly less power in the alpha band in the control asynchronous condition. Less power in the alpha band (i.e. greater suppression) is thought to reflect increased neural activity, thus neural activation in these regions was greater in the asynchronous condition than in the illusion condition. The stronger activation during asynchronous stroking may have resulted from the detection of greater visuo-tactile conflict. The data from the present study show that the later SEP map was enhanced and prolonged in strength during the asynchronous condition compared to the synchronous condition. This SEP map was also longer in duration than the earlier map (map 2). Our data are thus shown to be complementary to those of this earlier EEG study because the frequency analysis carried out in the latter did not distinguish between the durations of different activations. The present study alone (fMRI cannot make this distinction either) is able to functionally dissociate between an earlier activation in SI and a later activation in the same and/or higher tier areas as it shows they are differently modulated by the synchrony manipulation.

In addition to the findings of FBI research, synchronous visuo-tactile stimulation has also been linked to changes in somatosensory cortex by two RHI studies (Schaefer et al., 2006; Press et al., 2008). For example, (Press et al., 2008) showed that a late negative SEP component (at a similar time period to map 5 , at $\sim 140 \mathrm{~ms}$ ) to tactile taps was enhanced after a training period of synchronous stroking compared to asynchronous stroking. However, there were several differences between this study and the present study, apart from it being a body part illusion, not a FBI: thus, the SEPs in the RHI study were recorded after a 'training' period of stroking whereas
SEPs in the present study were recorded during the stroking period.

Finally, we note that it has been shown (Cardini et al., 2011; Longo et al., 2011) that vision of the body results in a reduction in the amplitude of SEPs (at 27-50 ms after electrical stimulation of the median nerve, compared to viewing an object), and viewing pain and tactile stimuli delivered to another's body modulates the amplitude of the P45 SEP component (Bufalari et al., 2007). Vision of the body cannot however explain the present effects, as our comparison was between synchronous and asynchronous stroking conditions; participants viewed their body in both.

\section{Attentional modulation}

It is also possible that the earlier SEP enhancement is - at least partly - due to differences in attention between the illusion and the control condition. Some previous SEP studies have shown that relatively early SEP components (e.g. P27, P50) are modulated by attention (Josiassen et al., 1982; Seitz and Roland, 1992; Kunde and Treede, 1993; Mima et al., 1998) and one study (Legon and Staines, 2006) showed modulation of the N20 (to medial nerve stimulation) when attention to a tactile stimulus is needed to guide a motor task. Other studies have failed to find early attention effects (Desmedt and Robertson, 1977; Zopf et al., 2004). Attentional effects at the latency of our later component have also been found: there is evidence that brain activity during this later time period reflects relatively complex aspects of tactile processing, e.g. these components are classically modulated by attention (Desmedt and Robertson, 1977; Josiassen et al., 1982; Garcia-Larrea et al., 1995; Mima et al., 1998). There is also evidence that mid-latency components are also dependent on conscious awareness (Schubert et al., 2006): in a study of backward masking the P100 and N140 SEP components had larger amplitude when the tactile targets were consciously perceived.

It is conceivable that the $\mathrm{FBI}$ may induce differences in spatial attention between the synchronous and asynchronous conditions. Since the illusion involves mislocalisation of tactile stimuli to the virtual body and a shift in self-location towards it, it may also induce a shift in spatial attention to far space in which the virtual body is viewed. In the asynchronous condition there is weaker tactile mislocalisation and shift in self-location and possibly also a weaker shift in spatial attention from personal to far (extrapersonal) space. Several studies have suggested that the human and non-human primate brain encodes personal, peripersonal and far space differently (e.g. (Rizzolatti et al., 1985; Rizzolatti and Camarda, 1987; Halligan and Marshall, 1991; Vuilleumier et al., 1998; Ortigue et al., 2006)). Moreover, attending to near vs. far space has been demonstrated to employ either ventral or dorsal visual areas, respectively (Weiss et al., 2000). Shifting attention between near and far spaces has also been shown to have behavioural costs compared to shifting attention within the same space (Couyoumdjian et al., 2003; Ferlazzo et al., 2008). It is therefore conceivable that there could also be EP differences due to orienting 
spatial attention to different spaces and that this could relate to the early SEP amplitude differences we observe in the present study.

\section{Functional deafferentation}

Although we did not directly question participants about a specific feeling of disownership of their physical bodies during the illusion, it is possible that increased ownership for the virtual body (self-identification) was accompanied by decreased ownership for their physical body and that this may be associated with the SEP enhancement we see during the illusion for the earlier component as we discuss below. Decreased ownership for the physical body is also compatible with findings in a recent study. Thus, (Hänsel et al., 2011) reported analgesic effects characterised by increased pain thresholds during the $\mathrm{FBI}$, compatible with functional deafferentation during the illusion. Also, a recent SEP study (Dieguez et al., 2009) showed that the first component of median nerve SEPs was enhanced in contralateral SI during an illusory finger sensation of numbness. Other findings in the related $\mathrm{RHI}$ also revealed physiological data that are compatible with functional deafferentation (Moseley et al., 2008). The physiological changes may be due to increased disownership affecting homeostatic control of the disowned body/body part (Moseley et al., 2012).

It has previously been shown that actual physical deafferentation leads to an SEP enhancement. The latter has been observed during local anaesthesia (Tinazzi et al., 1997) and ischaemic nerve block (Werhahn et al., 2002), two conditions that have also been associated with loss of ownership (Paqueron et al., 2003). Evidence that body illusions of ownership can produce similar behavioural and neural effects to those produced by deafferentation also come from a recent SEP study (Dieguez et al., 2009) which showed that the first component of median nerve SEPs was enhanced in contralateral SI during an illusory finger sensation. Although there are data from diverse paradigms supporting the hypothesis that functional deafferentation may enhance somatosensory cortical processing, this can only be a tentatively suggested explanation for the present data since we did not measure the participants sense of disownership nor physiological measures of deafferentation in the present study.

\section{Map duration}

Our discussion has focussed on SEP amplitude differences but for the later map 5 we also found a difference in duration: this microstate lasted significantly longer in the asynchronous than in the synchronous condition. There were no differences in map duration for map 2. Prolonged brain activity may be a consequence of re-entrant input via top-down connections (David et al., 2005). Alternatively, it may be caused by a change in functional connectivity (Friston and Frith, 1995), although this would be more likely to change map topography (Lehmann et al., 1987). We therefore speculate that the duration of the microstate underlying map 5 was longer in the asynchronous condition due to a difference in the top-down input from multisensory areas (which integrate visuo-tactile signals) to somatosensory cortex.

\section{CONCLUSIONS}

The present findings show that experimentally induced changes in self-identification with a virtual body modulates activity in primary somatosensory cortex as well as in higher-tier parietal (and possibly frontal) cortex. Based on the timing of these activations and the experimentally induced changes in illusory self-identification we argue that the early activity changes in primary somatosensory cortex may be a consequence of (1) multisensory integration of congruent vs. incongruent visuo-tactile stimuli and/ or (2) differences in spatial attention (to near or far space) between the synchronous and asynchronous conditions. Finally, we suggest that the later activation in higher-tier parietal cortex (and potentially other regions in temporoparietal and frontal cortex) reflects the detection of visuo-tactile conflicts/mismatches in the asynchronous condition.

Acknowledgments-This study was supported by the 'NestecEPFL Alliance'. We thank T. Lavanchy for creating Figure 1. We thank two anonymous reviewers for their valuable input.

\section{REFERENCES}

Abbruzzese G, Abbruzzese M, Favale E, Ivaldi M, Leandri M, Ratto S (1980) The effect of hand muscle vibration on the somatosensory evoked potential in man: an interaction between lemniscal and spinocerebellar inputs? J Neurol Neurosurg Psychiatry 43:433-437.

Allison T, McCarthy G, Wood CC, Darcey TM, Spencer DD, Williamson PD (1989) Human cortical potentials evoked by stimulation of the median nerve. I. Cytoarchitectonic areas generating short-latency activity. J Neurophysiol 62:694-710.

Allison T, McCarthy G, Wood CC, Jones SJ (1991) Potentials evoked in human and monkey cerebral cortex by stimulation of the median nerve: a review of scalp and intracranial recordings. Brain 114:2465-2503.

Allison T, McCarthy G, Wood CC, Williamson PD, Spencer DD (1989) Human cortical potentials evoked by stimulation of the median nerve. II. Cytoarchitectonic areas generating long-latency activity. $\mathrm{J}$ Neurophysiol 62:711-722.

Arzy S, Thut G, Mohr C, Michel CM, Blanke O (2006) Neural basis of embodiment: distinct contributions of temporoparietal junction and extrastriate body area. J Neurosci 26:8074-8081.

Asanuma K, Urushihara R, Nakamura K, Kitaoka K, Sei H, Morita Y, Shibasaki H, Kaji R (2003) Premovement gating of somatosensory evoked potentials after tibial nerve stimulation. Neuroreport 14:375-379.

Aspell JE, Lenggenhager B, Blanke O (2009) Keeping in touch with one's self: multisensory mechanisms of self-consciousness. PLoS ONE 4:e6488.

Baumgärtner U, Vogel H, Ellrich J, Gawehn J, Stoeter P, Treede RD (1998) Brain electrical source analysis of primary cortical components of the tibial nerve somatosensory evoked potential using regional sources. Electroencephalogr Clin Neurophysiol/ Evoked Potentials Section 108:588-599.

Blanke O, Metzinger T (2009) Full-body illusions and minimal phenomenal selfhood. Trends Cogn Sci 13:7-13.

Blanke O, Mohr C, Michel C, Pascual-Leone A, Brugger P, Seeck M, Landis T, Thut G (2005) Linking out-of-body experience and self processing to mental own-body imagery at the temporoparietal junction. J Neurosci 25:550-557. 
Botvinick M, Cohen J (1998) Rubber hands 'feel' touch that eyes see. Nature 391:756.

Bremmer F, Schlack A, Duhamel J-R, Graf W, Fink GR (2001) Space coding in primate posterior parietal cortex. Neuroimage 14:S46-S51.

Bufalari I, Aprile T, Avenanti A, Di Russo F, Aglioti SM (2007) Empathy for pain and touch in the human somatosensory cortex. Cereb Cortex 17:2553-2561.

Cardini F, Longo MR, Haggard P (2011) Vision of the body modulates somatosensory intracortical inhibition. Cereb Cortex 21: 2014-2022.

Caselli RJ (1993) Ventrolateral and dorsomedial somatosensory association cortex damage produces distinct somesthetic syndromes in humans. Neurology 43:762-771.

Casey KL, Minoshima S, Berger KL, Koeppe RA, Morrow TJ, Frey KA (1994) Positron emission tomographic analysis of cerebral structures activated specifically by repetitive noxious heat stimuli. J Neurophysiol 71:802-807.

Chiappa $\mathrm{K}$ Evoked potentials in clinical medicine. 3rd ed. Philadelphia, PA: Lippincott-Raven; 1997.

Corbetta M, Shulman GL (2002) Control of goal-directed and stimulus-driven attention in the brain. Nat Rev Neurosci 3:201-215

Cruse R, Klem G, Lesser RP, Lueders H (1982) Paradoxical lateralization of cortical potentials evoked by stimulation of posterior tibial nerve. Arch Neurol 39:222-225.

David O, Harrison L, Friston KJ (2005) Modelling event-related responses in the brain. Neuroimage 25:756-770.

Desmedt JE, Bourguet M (1985) Color imaging of parietal and frontal somatosensory potential fields evoked by stimulation of median or posterior tibial nerve in man. Electroencephalogr Clin Neurophysiol 62:1-17.

Desmedt JE, Robertson D (1977) Differential enhancement of early and late components of the cerebral somatosensory evoked potentials during forced-paced cognitive tasks in man. J Physiol 271:761-782.

Desmedt JE, Tomberg C (1989) Mapping early somatosensory evoked potentials in selective attention: critical evaluation of control conditions used for titrating by difference the cognitive P30, P40, P100 and N140. Electroencephalogr Clin Neurophysiol/ Evoked Potentials Section 74:321-346.

Dieguez S, Mercier MR, Newby N, Blanke O (2009) Feeling numbness for someone else's finger. Curr Biol 19:R1108-R1109.

Downar J, Crawley AP, Mikulis DJ, Davis KD (2000) A multimodal cortical network for the detection of changes in the sensory environment. Nat Neurosci 3:277-283.

Ehrsson H (2007) The experimental induction of out-of-body experiences. Science 317:1048.

Ehrsson H, Spence C, Passingham R (2004) That's my hand! Activity in premotor cortex reflects feeling of ownership of a limb. Science 305:875-877.

Ehrsson HH, Holmes NP, Passingham RE (2005) Touching a rubber hand: feeling of body ownership is associated with activity in multisensory brain areas. J Neurosci 25:10564-10573.

Ehrsson $\mathrm{HH}$, Wiech $\mathrm{K}$, Weiskopf $\mathrm{N}$, Dolan RJ, Passingham RE (2007) Threatening a rubber hand that you feel is yours elicits a cortical anxiety response. Proceedings of the National Academy of Sciences 104:9828-9833.

Eimer M, Cockburn D, Smedley B, Driver J (2001) Cross-modal links in endogenous spatial attention are mediated by common external locations: evidence from event-related brain potentials. Exp Brain Res 139:398-411.

Eimer M, Driver J (2001) Crossmodal links in endogenous and exogenous spatial attention: evidence from event-related brain potential studies. Neurosci Biobehav Rev 25:497-511.

Ferri R, Del Gracco S, Elia M, Musumeci SA, Spada R, Stefanini MC (1996) Scalp topographic mapping of middle-latency somatosensory evoked potentials in normal aging and dementia. Neurophysiol Clin 26:311-319.

Ferlazzo F, Fagioli S, Di Nocera F, Sdoia S (2008) Shifting attention across near and far spaces: implications for the use of hands-free cell phones while driving. Accident, Analysis and Prevention 40:1859-1864.

Folstein JR, Van Petten C (2008) Influence of cognitive control and mismatch on the N2 component of the ERP: a review. Psychophysiology 45:152-170.

Forss N, Hari R, Salmelin R, Ahonen A, Hämäläinen M, Kajola M, Knuutila J, Simola J (1994) Activation of the human posterior parietal cortex by median nerve stimulation. Exp Brain Res 99:309-315.

Forss N, Merlet I, Vanni S, Ha "ma"la" inen M, Mauguie're Fo, Hari R (1996) Activation of human mesial cortex during somatosensory target detection task. Brain Res 734:229-235.

Friston KJ, Frith CD (1995) Schizophrenia a disconnection syndrome. Clini Neurosci (Newyork, NY) 3:89-97.

Gallagher S How the body shapes the mind. Oxford: Clarendon Press; 2005.

Garcia-Larrea L, Lukaszewicz A-C, Mauguiere F (1995) Somatosensory responses during selective spatial attention: the N120-to-N140 transition. Psychophysiology 32:526-537.

Gardill K, Hielscher H (2001) Multichannel derived median nerve SEP compared to EEG in patients with vascular cerebral lesions. Electromyogr Clin Neurophysiol 41:215-223.

Gobbelé R, Waberski TD, Thyerlei D, Thissen M, Darvas F, Klostermann F, Curio G, Buchner H (2003) Functional dissociation of a subcortical and cortical component of highfrequency oscillations in human somatosensory evoked potentials by motor interference. Neurosci Lett 350:97-100.

Halligan PW, Marshall JC (1991) Left neglect for near but not far space in man. Nature 350:498-500.

Hänsel $A$, Lenggenhager $B$, von Känel $R$, Curatolo $M$, Blanke $O$ (2011) Seeing and identifying with a virtual body decreases pain perception. Eur J Pain 15:874-879.

Heed T, Habets B, Sebanz N, Knoblich G (2010) Others' actions reduce crossmodal integration in peripersonal space. Curr Biol 20:1345-1349.

Hume AL, Cant BR (1978) Conduction time in central somatosensory pathways in man. Electroencaphalogr Clin Neurophysiol 45:361-375.

Ionta S, Heydrich L, Lenggenhager B, Mouthon M, Fornari E, Chapuis D, Gassert R, Blanke O (2011) Multisensory mechanisms in temporo-parietal cortex support self-location and first-person perspective. Neuron 70:363-374.

Jeannerod M (2007) Being oneself. J Physiol-Paris 101:161-168.

Jones SJ, Small DG (1978) Spinal and sub-cortical evoked potentials following stimulation of the posterior tibial nerve in man. Electroencephalogr Clin Neurophysiol 44:299-306.

Josiassen RC, Shagass C, Roemer RA, Ercegovac DV, Straumanis JJ (1982) Somatosensory evoked potential changes with a selective attention task. Psychophysiology 19:146-159.

Kakigi R, Koyama S, Hoshiyama M, Shimojo M, Kitamura Y, Watanabe S (1995) Topography of somatosensory evoked magnetic fields following posterior tibial nerve stimulation. Electroencephalogr Clin Neurophysiol 95:127-134.

Kakigi R, Shibasaki H, Hashizume A, Kuroiwa Y (1982) Short latency somatosensory evoked spinal and scalp-recorded potentials following posterior tibial nerve stimulation in man. Electroencephalogr Clin Neurophysiol 53:602-611.

Kanayama N, Sato A, Ohira H (2007) Crossmodal effect with rubber hand illusion and gamma-band activity. Psychophysiology 44:392-402.

Kanayama N, Sato A, Ohira H (2009) The role of gamma band oscillations and synchrony on rubber hand illusion and crossmodal integration. Brain Cogn 69:19-29.

Kany C, Treede RD (1997) Median and tibial nerve somatosensory evoked potentials: middle-latency components from the vicinity of the secondary somatosensory cortex in humans. Electroencephalogr Clin Neurophysiol/Evoked Potentials Section 104:402-410.

Kida T, Nishihira Y, Wasaka T, Sakajiri Y, Tazoe T (2004) Differential modulation of the short- and long-latency somatosensory evoked potentials in a forewarned reaction time task. Clini Neurophysiol 115:2223-2230. 
Kunde V, Treede RD (1993) Topography of middle-latency somatosensory evoked potentials following painful laser stimuli and non-painful electrical stimuli. Electroencephalogr Clin Neurophysiol - Evoked Potentials 88:280-289.

Lascano AM, Brodbeck V, Lalive PH, Chofflon M, Seeck M, Michel CM (2009) Increasing the diagnostic value of evoked potentials in multiple sclerosis by quantitative topographic analysis of multichannel recordings. J Clin Neurophysiol 26:316-325. 310.1097/WNP.1090b1013e3181baac1000.2009.

Legon W, Staines WR (2006) Predictability of the target stimulus for sensory-guided movement modulates early somatosensory cortical potentials. Clin Neurophysiol 117:1345-1353.

Lehmann D, Ozaki H, Pal I (1987) EEG alpha map series: brain micro-states by space-oriented adaptive segmentation. Electroencephalogr Clin Neurophysiol 67:271-288.

Lehmann D, Skrandies W (1980) Reference-free identification of components of checkerboard-evoked multichannel potential fields. Electroencephalogr Clin Neurophysiol 48:609-621.

Lenggenhager B, Halje P, Blanke O (2011) Alpha band oscillations correlate with illusory self-location induced by virtual reality. Eur J Neurosci 2011.

Lenggenhager B, Mouthon M, Blanke O (2009) Spatial aspects of bodily self-consciousness. Conscious Cogn 18:110-117.

Lenggenhager B, Tadi T, Metzinger T, Blanke O (2007) Video ergo sum: manipulating bodily self-consciousness. Science 317:1096-1099.

Longo MR, Pernigo S, Haggard P (2011) Vision of the body modulates processing in primary somatosensory cortex. Neurosci Lett 489:159-163.

Lopez C, Mercier MR, Halje P, Blanke O (2011) Spatiotemporal dynamics of visual vertical judgments: early and late brain mechanisms as revealed by high-density electrical neuroimaging. Neuroscience 181:134-149.

Macaluso E, Driver J (2005) Multisensory spatial interactions: a window onto functional integration in the human brain. Trends Neurosci 28:264-271.

Macaluso E, Maravita A (2010) The representation of space near the body through touch and vision. Neuropsychologia 48:782-795.

Mercier MR, Schwartz S, Michel C, Blanke O (2009) Motion direction tuning in human visual cortex. Eur J Neurosci 29:424-434.

Michel CM, Murray MM (2012) Towards the utilization of EEG as a brain imaging tool. Neuroimage 61:371-385.

Michel CM, Thut G, Morand S, Khateb A, Pegna AJ, Grave de Peralta R, Gonzalez S, Seeck M, Landis T (2001) Electric source imaging of human brain functions. Brain Res Rev 36:108-118.

Mima T, Nagamine T, Nakamura K, Shibasaki H (1998) Attention modulates both primary and second somatosensory cortical activities in humans: a magnetoencephalographic study. J Neurophysiol 80:2215-2221.

Miura T, Sonoo M, Shimizu T (2003) Establishment of standard values for the latency, interval and amplitude parameters of tibial nerve somatosensory evoked potentials (SEPs). Clin Neurophysiol 114:1367-1378.

Moseley GL, Gallace A, Spence C (2012) Bodily illusions in health and disease: physiological and clinical perspectives and the concept of a cortical 'body matrix'. Neurosci Biobehav Rev 36:34-46.

Moseley GL, Olthof N, Venema A, Don S, Wijers M, Gallace A, Spence C (2008) Psychologically induced cooling of a specific body part caused by the illusory ownership of an artificial counterpart. Proceedings of the National Academy of Sciences 105:13169-13173.

Mountcastle VB Central nervous system mechanisms in the mechanoreceptive sensibility. In: Plum F, editor. Handbook of physiology. Bethesda, MD: American Psychological Association; 1984. p. 789-878

Murray M, Brunet D, Michel C (2008) Topographic ERP analyses: a step-by-step tutorial review. Brain Topogr 20:249-264.

Näätänen R (1975) Selective attention and evoked potentials in humans - a critical review. Biol Psychol 2:237-307.
Näätänen R, Gaillard AWK, Mäntysalo S (1978) Early selectiveattention effect on evoked potential reinterpreted. Acta Psychol 42:313-329.

Ortigue S, Mégevand P, Perren F, Landis T, Blanke O (2006) Double dissociation between representational personal and extrapersonal neglect. Neurology 66:1414-1417.

Palluel E, Aspell JE, Lavanchy T, Blanke O (2012) Experimental changes in bodily self-consciousness are tuned to the frequency sensitivity of proprioceptive fibers. Neuroreport 23:354-359.

Palluel E, Aspell JE, Blanke O (2011) Leg muscle vibration modulates bodily self-consciousness: integration of proprioceptive, visual, and tactile signals. J Neurophysiol 105:2239-2247.

Paqueron X, Leguen M, Rosenthal D, Coriat P, Willer JC, Danziger N (2003) The phenomenology of body image distortions induced by regional anaesthesia. Brain 126:702-712.

Pavani F, Spence C, Driver J (2000) Visual capture of touch: out-ofthe-body experiences with rubber gloves. Psychol Sci 11:353-359.

Petkova V, Ehrsson H (2009) When right feels left: referral of touch and ownership between the hands. PloS ONE 4:e6933.

Petkova Valeria I, Björnsdotter M, Gentile G, Jonsson T, Li T-Q, Ehrsson HH (2011) From part- to whole-body ownership in the multisensory brain. Curr Biol 21:1118-1122.

Press C, Heyes C, Haggard P, Eimer M (2008) Visuotactile learning and body representation: an ERP study with rubber hands and rubber objects. J Cogn Neurosci 20:312-323.

Rizzolatti G, Camarda R Neural circuits for spatial attention and unilateral neglect. In: Jeannerod $M$, editor. Neurophysiological and neuropsychological aspects of spatial neglect. North Holland: Amsterdam; 1987. p. 151-183.

Rizzolatti G, Gentilucci M, Matelli M Selective spatial attention: one center, one circuit, or many circuits? In: Posner M, Marin $\mathrm{O}$, editors. Attention and performance II. Hillsdale: Lawrence Erlbaum Associates; 1985. p. 251-265.

Rossini PM, Caramia D, Bassetti MA, Pasqualetti P, Tecchio F, Bernardi G (1996) Somatosensory evoked potentials during the ideation and execution of individual finger movements. Muscle Nerve 19:191-202.

Sathian K, Stilla R (2010) Cross-modal plasticity of tactile perception in blindness. Restor Neurol Neurosci 28:271-281.

Schaefer M, Noennig N, Heinze H-J, Rotte M (2006) Fooling your feelings: artificially induced referred sensations are linked to a modulation of the primary somatosensory cortex. Neuroimage 29:67-73.

Schubert R, Blankenburg F, Lemm S, Villringer A, Curio G (2006) Now you feel it—now you don't: ERP correlates of somatosensory awareness. Psychophysiology 43:31-40.

Schwabe L, Blanke O (2008) The vestibular component in out-ofbody experiences: a computational approach. Front Hum Neurosci 2:17.

Schwabe L, Lenggenhager B, Blanke, (2009) The timing of temporoparietal and frontal activations during mental own body transformations from different visuospatial perspectives. Human brain mapping 30:1801-1812.

Seitz RJ, Roland PE (1992) Vibratory stimulation increases and decreases the regional cerebral blood flow and oxidative metabolism: a positron emission tomography (PET) study. Acta Neurol Scand 86:60-67.

Seyal M, Emerson RG, Pedley TA (1983) Spinal and early scalprecorded components of the somatosensory evoked potential following stimulation of the posterior tibial nerve. Electroencephalogr Clin Neurophysiol 55:320-330.

Slater M, Perez-Marcos D, Ehrsson H, Sanchez-Vives M (2008) Towards a digital body: the virtual arm illusion. Front Hum Neurosci 2.

Spence C, Pavani F, Driver J (2004) Spatial constraints on visualtactile cross-modal distractor congruency effects. Cogn Affect Behav Neurosci 4:148-169.

Taylor-Clarke M, Kennett S, Haggard P (2002) Vision modulates somatosensory cortical processing. Curr Biol 12:233-236. 
Tibshirani R, Walther G (2005) Cluster validation by prediction strength. J Comput Graphical Stat 14:511-528.

Tinazzi M, Zanette G, Polo A, Volpato D, Manganotti P, Bonato C, Testoni R, Fiaschi A (1997) Transient deafferentation in humans induces rapid modulation of primary sensory cortex not associated with subcortical changes: a somatosensory evoked potential study. Neurosci Lett 223:21-24.

Tsakiris M, Costantini M, Haggard P (2008) The role of the right temporo-parietal junction in maintaining a coherent sense of one's body. Neuropsychologia 46:3014-3018.

Tsakiris M, Hesse M, Boy C, Haggard P, Fink GR (2007) Neural signatures of body ownership: a sensory network for bodily selfconsciousness. Cereb Cortex 17:2235-2244.

Valeriani M, Restuccia D, Di Lazzaro V, Barba C, Le Pera D, Tonali P (1998) Dissociation induced by voluntary movement between two different components of the centro-parietal P40 SEP to tibial nerve stimulation. Electroencephalogr Clin Neurophysiol/Evoked Potentials Section 108:190-198. van de Wassenberg W, van der Hoeven J, Leenders K, Maurits N (2008) Multichannel recording of median nerve somatosensory evoked potentials. Neurophysiol Clin 38:9-21.

Vuilleumier P, Valenza N, Mayer E, Reverdin A, Landis T (1998) Near and far visual space in unilateral neglect. Ann Neurol 43:406-410.

Weiss PH, Marshall JC, Wunderlich G, Tellmann L, Halligan PW, Freund H-J, Zilles K, Fink GR (2000) Neural consequences of acting in near versus far space. a physiological basis for clinical dissociations. Brain 123:2531-2541.

Werhahn KJ, Mortensen J, Van Boven RW, Zeuner KE, Cohen LG (2002) Enhanced tactile spatial acuity and cortical processing during acute hand deafferentation. Nat Neurosci 5:936-938.

Zopf R, Giabbiconi CM, Gruber T, Müller MM (2004) Attentional modulation of the human somatosensory evoked potential in a trial-by-trial spatial cueing and sustained spatial attention task measured with high density 128 channels EEG. Cogn Brain Res 20:491-509. 